\title{
CONFLICTOS URBANÍSTICOS Y MOVILIZACIONES CIUDADANAS: REFLEXIONES DESDE BARCELONA
}

\author{
Helena CRUZ i Gallach ${ }^{1}$ \\ MARC MARTí-Costa ${ }^{2}$
}

\begin{abstract}
Resumen - El artículo propone, de un lado, analizar cómo la evolución del planeamiento urbanístico en un contexto de globalización ha facilitado el surgimiento de nuevos conflictos urbanísticos y, de otro, identificar cómo se articulan las variadas formas de movilización ciudadana en relación a estos conflictos. Finalmente, a través del caso de la defensa del complejo industrial de Can Ricart de Barcelona se ejemplifica cómo las plataformas ciudadanas consiguen poner en entredicho el modelo de desarrollo urbano de la ciudad.
\end{abstract}

Palabras clave: Conflicto urbanístico, plan urbanístico, plataformas ciudadanas, movilizaciones sociales, Barcelona.

Resumo - CONFlitos URBANOS E MOBILIZAÇÃo DE CIDADÃos: REFLEXõES A PARTIR DE BARCELONA. Este artigo analisa, por um lado, a evolução do planeamento urbano num contexto de globalização, o qual facilitou o surgimento de conflitos urbanos. Por outro, identifica as várias formas de mobilização dos cidadãos em relação a estes conflitos. Finalmente, através do caso originado pela defesa do património industrial de Can Ricart, em Barcelona, exemplifica-se como as plataformas cidadãs questionam o modelo de desenvolvimento urbano.

Palavras-chave: Conflito urbano, plano urbanístico, plataformas cidadãs, mobilizações sociais, Barcelona.

Abstract - CONFLICTS OVER URBAN PLANNING AND CITIZENS' MOBILISATION:
REFLECTIONS ON BARCELONA. The aim of this article is two-fold: to analyse how the
evolution of urban planning in a context of globalisation has facilitated the emergence
of conflicts over urban planning and to identify how the various forms of social
mobilisation are interconnected. Specifically, the case of Can Ricart in Barcelona is

Recebido: 10/02/2010. Aceite: 06/09/2010.

1 Departament de Geografia. Universitat Autònoma de Barcelona. E-mail: Helena.Cruz@, uab.cat

2 Institut de Govern i Polítiques Públiques. Universitat Autònoma de Barcelona. Escola de Polítiques Socials i Urbanes. E-mail: Marc.Marti@uab.cat 
discussed in order to exemplify how citizens' committees are able to question the city's urban development model.

Keywords: Urban planning conflict, urban plan, citizens' committees, social mobilisation, Barcelona.

Résumé - CONFLITS URBAINS ET MOBILISATIONS CITOYENNES. QUELQUES RÉFLEXIONS À PARTIR DE BARCELONE. On montre comment l'évolution de la planification urbaine, dans un contexte de mondialisation, a fait surgir de nouveaux conflits urbains et comment les diverses formes de mobilisation citoyenne s'articulent par rapport à ces conflits. En s'appuyant sur le cas de la défense du complexe industriel de Can Ricart, à Barcelone, on montre finalement comment les plateformes citoyennes peuvent parvenir à mettre en cause le modèle de développement urbain de la ville.

Mots clés: Conflit d'aménagement, plan urbanistique, plateforme citoyenne, mobilisations sociales, Barcelone.

\section{INTRODUCCIÓN}

El proceso de desvirtuación que ha sufrido el plan urbanístico durante las últimas décadas, fomentado por la predilección por otras formas de planificación del territorio como el planeamiento estratégico o la actuación mediante proyectos urbanos, ha sido influenciado por un contexto de globalización que ha llevado a las ciudades a un espiral de competencia ascendente. En consecuencia, se han limitado los espacios de participación ciudadana durante el proceso de planificación urbana y éste se ha tecnificado y restringido sólo a determinadas elites. El artículo analiza cómo este contexto ha propiciado el estallido de conflictos urbanísticos y, de otro lado, evidencia las nuevas formas que están tomando las movilizaciones ciudadanas, todo ello ejemplificándolo con las transformaciones urbanísticas del barrio de Poblenou de Barcelona. Si bien la ciudad de Barcelona ya gozó, durante los años sesenta y setenta, de una amplia movilización ciudadana que radicó en la creación de las asociaciones de vecinos, en la última década han surgido nuevos conflictos urbanísticos y se han diversificado las formas de organización de las protestas ciudadanas.

Entendemos los conflictos como estados de tensión en la relación entre las administraciones y los ciudadanos que estallan cuando los objetivos y propósitos de ambos actores no convergen, creándose una condición de revuelta que se materializa con la organización de plataformas cívicas o asociaciones. De esta manera, los ciudadanos reivindican la necesidad de afrontar y resolver problemáticas urbanas o bien se oponen a proyectos que incomodan o amenazan al propio territorio. Ya sea con un rol de propuesta o de contraposición, reclaman poder incidir en la toma de decisiones que les incumben. Además, cuando consiguen una movilización creciente, se convierten en un instrumento de presión hacia la opinión pública creando cierto malestar a los entornos políticos (Lagomarsino, 2002). 
En primer lugar, el artículo ejemplifica cómo la evolución que ha sufrido el planeamiento urbanístico y el gobierno del territorio en general durante las últimas décadas, al ponerse al servicio de los intereses globales, ha comportado una limitación de los canales de participación ciudadana como espacios de debate y presentación de alternativas y ha facilitado la proliferación de los conflictos urbanísticos. Seguidamente se presentan algunas de las dinámicas que han facilitado la aparición de nuevas movilizaciones sociales. En el tercer apartado, focalizamos en las estrategias que desarrollan los actores que toman parte en el conflicto: ¿qué diferencias y similitudes podemos establecer entre los distintos colectivos (plataformas ciudadanas, asociaciones de vecinos, entidades coordinadoras, etcétera)? Finalmente ejemplificamos el análisis presentado con el caso de Can Ricart, en el barrio del Poblenou de Barcelona.

\section{PLANEAMIENTO URBANÍSTICO Y PARTICIPACIÓN CIUDADANA}

\section{Globalización, metropolización y nuevas formas de planeamiento urbano}

La ciudad se caracteriza tanto por su complejidad como por estar en continua transformación. En este proceso de modificación constante, además, intervienen y participan diferentes agentes (propietarios del suelo, promotores inmobiliarios, medios de comunicación, políticos, técnicos, ciudadanos, asociaciones, grupos de presión, etc.) que presentan objetivos, deseos, intereses y opiniones diferentes, los cuales pueden llegar a ser antagónicos. Para hacer frente a esa complejidad, la acción de gobierno se dotó de instrumentos tales como el planeamiento, que escenifica en el plan el proyecto de futuro de una determinada ciudad, que "traduce bajo el aspecto físico y funcional la intencionalidad que la colectividad (a través de instrumentos democráticos) ha elaborado para su futuro" (Indovina, 2005: 52).

Frente a la diversidad de intereses existentes en el territorio, el plan urbanístico ha sido considerado como un documento de consenso, durante el cual los contrates e intereses divergentes se llevaban a situaciones de acuerdo. Para llegar a tal fin, la legislación española y catalana han previsto algunos procedimientos de debate entorno a los criterios y objetivos de los planes con la exposición pública de las propuestas $\mathrm{y}$, a veces, los ayuntamientos han organizado acciones complementarias tales como exposiciones, mesas redondas, publicaciones o talleres de participación (Esteban, 1999; Font, 2009). Al planeamiento urbanístico no le han faltado críticas por su tecnicismo y formalismo, por estar esencialmente atado a la lógica del desarrollo capitalista (Castells, 1976) o bien al servicio de un número muy limitado de actores (Logan y Molotoch, 1987). Así, la falta de participación de la ciudadanía en las decisiones urbanísticas y de ordenación del territorio ha sido una constante en su historia, a pesar de algunas corrientes surgidas en los países anglosajones y orientadas a mejorar las 
capacidades de incidencia de los habitantes (Davidoff, 1965; Alexander, 1976; Friedman, 1992; Healey, 1997), pero que no han tenido especial influencia en la disciplina urbanística en el Estado español.

Así pues, en el contexto español, aunque legislativamente se reservan espacios de participación para los diferentes agentes que toman parte del proceso de elaboración del planeamiento, en la práctica, demasiado a menudo la ciudadanía no puede establecer un diálogo con el equipo redactor. El proceso de participación se limita al período de exposición pública y a la redacción de alegaciones. Como consecuencia, no representa un espacio suficientemente abierto y deliberativo para que la ciudadanía pueda expresar sus opiniones, deseos, dudas o temores y, menos aún, para presentar alternativas. Esto ha propiciado el desbordamiento de los cauces establecidos por el planeamiento, la proliferación de los conflictos urbanísticos y la irrupción de movimientos sociales urbanos, ya que los ciudadanos ven en la protesta una vía para hacer sentir sus desazones y deseos e intentar incidir en el proceso de planificación.

Las transformaciones sociales y urbanas vinculadas al proceso de globalización y metropolización incorporan una mayor complejidad en el análisis de los conflictos urbanísticos, la participación ciudadana y los movimientos urbanos en el contexto español. Entre estas transformaciones, uno de los elementos más característicos es el renacimiento de lo local (Harvey, 1990; Castells, 1997; Bobbio, 1999; Nel·lo, 2003) el cual ha sido analizado a menudo por los estudios sobre la globalización y sobre la polarización global-local. Como resultado del proceso de globalización y del desarrollo de las nuevas tecnologías, la progresiva desaparición de las fronteras físicas, administrativas o de comunicación ha configurado unos territorios cada vez más integrados donde las características específicas de cada lugar devienen más importantes. Así pues, las singularidades locales renacen como incentivo para atraer a los flujos de inversión y potenciar sus especificidades competitivas (Harvey, 1990; Bobbio, 1999). Las oposiciones locales y el estallido de muchos conflictos territoriales estarían, en consecuencia, estrechamente relacionados con la irrupción de las identidades locales que nacen en contraposición a la tendencia de dominio de los flujos globales (Castells, 1997). Así, la proliferación de los conflictos relacionados con el territorio obedecen a la creciente preocupación de la población por la calidad, los recursos, y la seguridad del lugar donde viven (Nel·lo, 2003). Sin embargo, tesis como la de la reterritorialización (Brenner, 1999) o de la glocalización (Swyngedow, 1997) introducen perspectivas mucho más dialécticas y dinámicas en la relación unidireccional global-local. Así en la medida que las ciudades pasan a ser espacios "glocales" dónde confluyen múltiples escalas geográficas de forma potencialmente conflictiva, los movimientos urbanos se incorporarían también en esta dialéctica (Köhler y Wissen, 2003).

Este contexto ha influenciado también las formas tradicionales de gestión de las ciudades y la ejecución del planeamiento urbanístico en el Estado español, al ponerlos al servicio de los intereses globales. A raíz de la espiral competitiva en que han entrado las ciudades desde finales del siglo XX, persiguiendo la 
atracción de actividades económicas, habitantes y visitantes, los gobiernos han recorrido a técnicas e instrumentos de gestión originarios del mundo empresarial, como el marketing urbano o el planeamiento estratégico. Planteado en contraposición al planeamiento urbanístico tradicional, se presenta como un método de planificación horizontal que pretende concebir, con el consenso de los representantes de la administración pública y los principales agentes socioeconómicos, un futuro deseable para la ciudad (Forn y Pascual, 1995; Curti y Gibelli, 1996; Camagni, 2003; Cruz, 2006). Aunque se presenta como una alternativa más eficaz a la planificación urbanística, el planeamiento estratégico no contempla el territorio como referente principal. El diseño de la estructura urbana o la delimitación de las áreas de crecimiento y regeneración no son cuestiones clave para el planeamiento estratégico, que se centra en consensuar estrategias que buscan relanzar económicamente el municipio. Por otro lado, dedica sus esfuerzos a diagnosticar las amenazas, debilidades, fortalezas y oportunidades que presenta el municipio en cuestión y a la búsqueda de acuerdo sobre unos objetivos generales para afrontarlas (Ferrer y Sabaté, 1999). Además, el énfasis en su carácter participativo, y con una escenificación más rotunda del pacto, ha conllevado un alejamiento de los espacios de participación de los procesos de planificación urbanística.

Pero no sólo el planeamiento estratégico apareció como un instrumento atractivo para las administraciones públicas sino que el proyecto urbano también surgió desde finales de los años ochenta de forma relevante como método a través del cual actuar en las ciudades españolas. Este proceso se evidencia de una forma más relevante en las grandes ciudades y poco a poco se difunde como una nueva forma de gestión del territorio. A menudo se argumenta la necesidad de dotar el planeamiento urbanístico de una mejor capacidad de respuesta frente a los escenarios cambiantes. En este sentido, se busca una actuación urbanística rápida, flexible, puntual y eficaz, que pretende huir y evitar la rigidez y burocratización a la cual ha sido sometido el plan urbanístico. Con el tiempo, los proyectos urbanos han ganado envergadura hacia lo que se ha denominado megaproyectos, que tienen como características: a) son proyectos de escala metropolitana y regional, muy vinculados a la mejora de la competitividad de la región/metrópolis; b) habitualmente están relacionados con grandes infraestructuras con un alto potencial simbólico que implican procesos de renovación urbana en la área cercana; c) para su ejecución implican la colaboración de múltiples administraciones y empresas privadas que acaba cristalizando en la constitución de partenariados o consorcios.

En general, en nuestro país, el interés por el planeamiento como instrumento útil para la previsión y el control de las transformaciones territoriales se ha puesto en duda (Font, 2003). El desarrollo de nuevos proyectos puntuales y la elaboración de planes estratégicos paralelos conllevan una progresiva substitución del plan general, ya que tiene que absorber un continuo proceso de progresivos ajustes y modificaciones puntuales para ir incorporando los proyectos puntuales que surgen, hecho que para algunos ha llevado a "perder de vista la globalidad 
de la ciudad" (Navarro, 1999: 77). Estas tendencias han provocado que se haya desvirtuado el rol del plan como documento-intención de la colectividad urbana y ha comportado la elaboración de planes estratégicos difícilmente ejecutables debido a la inexistencia de un marco legislativo que los apoye. Como consecuencia, han proliferado las disputas para la localización de equipamientos o servicios, o para la ejecución de proyectos de regeneración urbana, hecho que nos ejemplifica como el planeamiento urbanístico no ha sido capaz de llevar a cabo su misión de establecer democráticamente un acuerdo general entorno a un proyecto de ciudad futura.

La ciudad de Barcelona inició ya a finales de los años ochenta - con la nominación a los Juegos Olímpicos - un proceso de renovación urbana profundo, ejecutado a través de distintas intervenciones parciales y en el marco de distintos planes estratégicos que perseguían el objetivo de internacionalizar la ciudad. La creación de la ciudad olímpica y los cinturones metropolitanos, pero especialmente la reforma del frente marítimo y del puerto marcaron el inicio de una política urbana no tan orientada al urbanismo de los barrios que había caracterizado el inicio de los años ochenta, sino hacia nuevas operaciones de mayor escala orientadas a modernizar la ciudad y relanzarla como una ciudad turística y de servicios. Una vez superada la resaca olímpica y la consolidación de las "nuevas áreas de centralidad" en el interior de la ciudad, se fortalece el desarrollo de la metrópolis a través de grandes proyectos metropolitanos, entre los que destaca el plan Delta (con la ampliación del puerto y del aeropuerto) y la planificación de la nueva estación del Tren de Alta Velocidad en la Sagrera. Además de las infraestructuras para afianzar Barcelona como centro logístico, se pone cada vez más el acento en la cultura y el conocimiento como nuevos motores de desarrollo de la ciudad. De todas formas, esto se materializa con grandes operaciones urbanísticas, como es la remodelación de la desembocadura del río Besòs con la celebración del Forum Universal de las Culturas 2004 y el "distrito de la innovación 22@bcn" que comentaremos más adelante. Son operaciones que, acompañadas de una política de marketing urbano y promoción económica significante, se han ido incorporando en el Plan General Metropolitano a través de modificaciones puntuales y se han dirigido a potenciar el rol internacional de la ciudad.

\section{La crisis de legitimidad, la protesta y la participación}

Aunque es evidente que la ubicación de una determinada instalación considerada desagradable o molesta tendrá más probabilidades de desarrollar un conflicto (Dear, 1992; Bobbio, 1999; Faggi y Turco, 2001; Nel·lo, 2003), a veces estallan porque sus promotores no establecen un diálogo previo a la aprobación del proyecto y no concuerdan su propuesta con los agentes urbanos. Así, en el Estado español, a menudo los conflictos aparecen a causa de la forma que toma el proceso de elaboración del planeamiento: la toma de decisión se lleva a cabo de forma cerrada en el ámbito meramente administrativo $y$, de esta forma, cuando 
se hace pública la decisión, no hace más que suscitar una reacción contraria por parte de aquellos que deben acoger el proyecto. El carácter jurídico-técnico del urbanismo no facilita la intervención de aquellos actores no expertos. Además, el proceso de elaboración y aprobación de los planes y proyectos urbanísticos a menudo no cuenta con mecanismos de participación ciudadana en las primeras fases de decisión. Así, el primer período de información pública se abre cuando el plan ya tiene establecidos sus objetivos y principales características, por lo que sólo aquellos actores con más recursos (por ejemplo para obtener información privilegiada) son capaces de ejercer presión.

En líneas generales, una de las causas del nacimiento de las movilizaciones ciudadanas entorno a cuestiones territoriales se debe a la desconfianza en las formas institucionales de representación ciudadana por parte de la población y en una cierta crisis de legitimidad del sistema representativo (Bobbio, 1999; Blanco y Gomà, 2002; Nel·lo, 2003; della Porta y Diani, 2004; Alfama et al., 2007). Subirats (2006) dibuja algunas de las problemáticas existentes:

«Resulta evidente la poca capacidad de resolución de los problemas que muestran los mecanismos democráticos de toma de decisiones. Formalismo, distanciamiento entre representantes y representados, opacidad, asimetría en los recursos de los teóricamente iguales... son algunas de las críticas que se esgrimen en el debate político dirigidas al funcionamiento actual de nuestros sistemas democráticos» (Subirats, 2006: 393).

Así, existe una conexión entre el auge de las nuevas formas de acción colectiva y las formas de representación política (Bobbio, 1999; della Porta y Diani, 2004). En este sentido, la proliferación de las movilizaciones ciudadanas es un signo de crisis de los instrumentos de articulación y agregación de intereses. Concretamente, su difusión responde al declive de la capacidad de los partidos de ser la conexión entre la sociedad civil y las instituciones, a la disminución del arraigo social de los partidos y la debilitación de su influencia en los procesos de decisión. En consecuencia, los ciudadanos se movilizan en base a las propias necesidades inmediatas y se dirigen directamente a la esfera pública con acciones de protesta para sensibilizar a los administradores y a la población en general (della Porta y Diani, 2004). La misma proliferación de este tipo de organización ciudadana acaba por "fragmentar ulteriormente la representación de los intereses y para hacer aún más difícil la tarea de los partidos en la mediación del impulso local" (Bobbio, 1999: 198). Una de las causas de este cambio surge del alejamiento de los centros de decisión respecto a la ciudadanía y de la burocratización y tecnificación de los procesos, que han tendido a hacer creer que las decisiones son imparciales y objetivas (Subirats, 2006).

En este contexto, los entornos políticos propician el nacimiento de nuevas formas de acción colectiva, de manera que los ciudadanos movilizados piden "contar más en las decisiones que los implican directa o indirectamente" (San- 
cassiani, 2005: 206). Pero en la planificación urbana, no es hasta en tiempos muy recientes que se popularizan algunos procesos y técnicas de participación que pretenden facilitar la implicación de la ciudadanía en las decisiones urbanísticas (Font, 2009), y se empiezan a regular por ley. Así van tomando fuerza procesos más plurales que ponen el acento en la colaboración y comunicación entre los diferentes actores implicados (Healey, 1997).

En el caso de Barcelona, en la última década el Ayuntamiento ha iniciado una serie de políticas de participación, aunque la ciudad ya contaba con algunos órganos, procesos y mecanismos (Ajuntament de Barcelona, 2009). En 2002 se aprobaron las Normas Reguladoras de Participación Ciudadana y progresivamente se implementaron nuevos órganos de participación territorial (consejos de distrito y consejos de barrio) pero también consejos sectoriales y audiencias públicas. La diagnosis sobre la participación ciudadana realizada para la elaboración del Plan Director Municipal de Participación Ciudadana 2010-2015 detectaba la existencia de un mapa complejo y poco sistematizado y coordinado de órganos, procesos y mecanismos de participación. Como consecuencia, revelaba que estos procesos conseguían un bajo impacto en las políticas, aunque también ponía de manifiesto que los ciudadanos a menudo desconocían la existencia de los procesos y mecanismos de participación (Ajuntament de Barcelona, 2009). Así pues, estas iniciativas municipales, con la elaboración de un Plan Director Municipal, muestran como durante los últimos años los esfuerzos se han dirigido a establecer nuevos espacios de participación, aunque al mismo tiempo no gocen de gran influencia.

La última propuesta sobre participación ciudadana y planificación urbana por parte del Ayuntamiento es el proceso participativo sobre la reforma de la parte central del eje viario de la Diagonal. Este proyecto, que plantea la reducción del espacio para vehículos y la posible introducción del tranvía en uno de los principales ejes viarios de la ciudad, ha sido escogido para acompañarlo con un proceso de participación ciudadana y se ha convertido en el estandarte de la política de participación municipal. Iniciado en febrero de 2009, el proceso prevé distintas sesiones formativas para dar a conocer el marco del proyecto y hacer comprensible el lenguaje urbanístico; la instauración de mesas sectoriales con las entidades para realizar aportaciones; la elaboración de dos alternativas por parte de la Oficina Técnica de la Diagonal; un posterior debate sobre éstas con jornadas informativas y exposiciones públicas; y, finalmente, una consulta ciudadana. En mayo de 2010 se realizó dicha consulta, que planteaba tres opciones: a) la creación de un boulevard; b) la creación de una rambla; c) ninguna de las anteriores. Con una participación del 12\% la opción ganadora fue la tercera con casi el $80 \%$ de los votos. Parece que esta opción acaparó distintas críticas: el desagrado hacia los proyectos planteados, el desacuerdo con la conveniencia de reformar la vía, la crítica hacia la administración que plateaba un proyecto con un importante gasto público y que era percibido como innecesario en un contexto de crisis económica, etcétera. La consulta ciudadana, que fue planteada como el gran proceso participativo de la ciudad, fue instrumentalizada por los distin- 
tos partidos políticos y se caracterizó por numerosos tropiezos técnicos, de manera que acabó por ser percibida como un fracaso lo que motivó el cese del primer teniente alcalde de la ciudad ${ }^{3}$.

La continuidad de los conflictos urbanísticos pone de manifiesto como diferentes valores e intereses están en juego y como existen diferentes proyectos de ciudad que han sido diseñados desde posicionamientos ideológicos contrapuestos. El modelo de crecimiento urbanístico promulgado por las elites económicas y políticas y el ayuntamiento las últimas décadas se ha basado en la especulación del suelo ${ }^{4}$ y en la orientación competitiva de la ciudad, mientras que otros actores - asociaciones de vecinos y entidades cívicas - han tendido a oponerse a este modelo reivindicando "el derecho a la ciudad", a través de la reclamación de servicios, equipamientos colectivos, vivienda asequible, espacios comunitarios de sociabilidad, etc. con el objetivo de mejorar la calidad de vida en los barrios. Sin embargo, este escenario se ha ido acomplejando con la aparición, por un lado, de algunas protestas desconectadas del interés general y, por el otro, por la existencia de actores en un mismo conflicto con diferentes intereses (tanto dentro del aparato político-administrativo como dentro el tejido social) que desdibujan la imagen simple de dos bloques contrapuestos.

\section{NUEVAS CONFIGURACIONES SOCIALES ENTORNO A LOS CONFLICTOS URBANÍSTICOS}

Seguidamente analizaremos algunos de los agentes que toman parte en los conflictos urbanísticos, cómo se organizan, qué rol adoptan y qué estrategias establecen para intentar modificar los proyectos urbanísticos. Partimos de la permisa que dados los cambios sociales y urbanos, existe una mayor fragmentación y multiplicidad de colectivos presentes, a la vez que se da una mayor interrelación entre temáticas urbanísticas, sociales, medioambientales y culturales en los conflictos. Tal y como afirman Martí-Costa y Bonet (2008a), constatamos que:

«No existe un único tipo de movimiento urbano. Simplificando mucho, ya no sólo abarcan cuestiones relacionadas con el consumo colectivo ni su principal actor son las asociaciones vecinales. Muchos de ellos expresan el legado de los nuevos movimientos sociales y de las luchas antiglobalizadoras; así como los cambios que han experimentado las propias ciudades. Hoy en día encontramos una gran heterogeneidad de colectivos, temáticas y formas de acción de grupos que actúan y politizan la ciudad». (Martí-Costa y Bonet, 2008a)

3 Para más información: http://www.ben.cat/diagonal

4 Por ejemplo, la administración planificó que la construcción de la nueva estación del tren de alta velocidad en Barcelona, la Sagrera, y la consiguiente remodelación del área se financiara a través de las plusvalías generadas por la recalificación del suelo y la venta de los terrenos. 
Así, en los conflictos urbanísticos a menudo "la oposición” no está promovida en primera persona por organizaciones preexistentes (administraciones públicas locales, partidos políticos, asociaciones cívicas o hasta ambientalistas) sino que está patrocinada por ciudadanos movilizados (Bobbio, 1999). Éstos se constituyen en actores colectivos organizándose en plataformas con el objetivo de ser un interlocutor para la administración. Precisamente, ciudadanos y determinadas asociaciones se unen en una plataforma para intentar modificar el plan en cuestión (introduciendo sus propuestas) o abolirlo. Por lo tanto, están destinados a disolverse una vez ha acabado la contienda, ya sea porque han conseguido influir en el contenido del plan o porque éste se ha redactado sin escuchar sus protestas. Las plataformas ejercen un rol de líder dentro de los grupos opositores y son aquellas que promueven la protesta ya que arraigan fácilmente en el tejido social. También es habitual que sean asociaciones preexistentes las que impulsen la constitución de coordinadoras o plataformas para facilitar el trabajo en red, aumentar su base social y su capacidad de presión.

En esta situación, las plataformas de ciudadanos han sido a menudo estigmatizadas como NIMBY (Not In My Back Yard, no en mi patio). Acrónimo inventado y difundido por autores norteamericanos, hace referencia a los movimientos que surgen como forma de resistencia delante la ubicación en su vecindad de infraestructuras, equipamientos o servicios que son considerados como incómodos, peligrosos o desagradables (della Porta, 2004). El síndrome NIMBY está asociado a un comportamiento conservador, a motivaciones egoístas, en el sentido de oposición al interés general (si el proyecto en cuestión se realizara en otra ubicación se aceptaría) y a acciones fragmentadas (Dear, 1992). Pero no en todos los conflictos se ejerce por parte de las plataformas una protesta basada en estos argumentos y, en muchos casos, más que la oposición a un determinado plan urbanístico con el objetivo de abolirlo, se pretende modificarlo con argumentos no estrictamente localistas.

Las plataformas de ciudadanos adoptan formas de coordinación flexibles y diversas entre ellas, pero la mayoría suelen tomar las decisiones en asamblea y actuar como grupos de presión. Si bien en muchos casos adoptan un carácter reactivo, ya que el objeto de su movilización es evitar el daño que creen que se derivaría de las decisiones que la administración pretende tomar, podemos decir que ese tipo de actitud es más bien característica de una fase inicial del conflicto donde el objetivo es la oposición. En algunos casos hemos detectado como, en fases más avanzadas del conflicto, las plataformas intentan revestir su discurso de valores más universales para legitimar su posición y acceden a avanzar en el terreno de la negociación. Así, a veces consiguen elaborar sus propios estudios, investigar y seguir las actividades urbanísticas de las administraciones, intercambian información y experiencias (ya sea con otras plataformas o asociaciones) e incorporan expertos a sus filas para que les asesoren y así fortalecer sus discursos y posición. Los ciudadanos que forman parte de estas plataformas a menudo están capacitados para contestar con argumentos de base técnica y científica, así como debatir con el lenguaje pertinente con los políticos y los 
técnicos municipales un determinado proyecto. Además, buscan en los medios de comunicación locales un aliado para expandir su ámbito de influencia y reforzarse como un actor más en el conflicto.

Las plataformas tienden a subrayar su distancia respecto al mundo de la política y remarcan su independencia aunque no dudan en utilizar las instituciones y partidos (simpatizantes) para conseguir influir en el proceso de toma de decisiones y modificar el proyecto. En este sentido, podemos decir que tienden a sustituir a las fuerzas políticas como única forma verdadera y legítima de representación de los intereses que se consideran perjudicados por las decisiones del gobierno local.

Barcelona cuenta con una larga tradición de asociacionismo. Durante los años setenta, las asociaciones de vecinos (de un bloque de viviendas, de una calle, de un barrio) fueron protagonistas en las relaciones con las administraciones locales como representantes de la ciudadanía. La dejadez que caracterizó la actuación urbanística y la falta de políticas sociales de las administraciones franquistas fue fuente de reivindicación por parte de la ciudadanía. Gracias a la Ley de Asociaciones de 1964 se constituyeron para reivindicar mejoras urbanas, el cumplimiento de la legislación urbanística, evitar la especulación y conseguir equipamientos y servicios. Representaron un espacio de confluencia de organizaciones sindicales, políticas, vecinales, etc., no sólo para mejorar la vida en los barrios sino como plataforma para reivindicar la democracia. A lo largo de estos años, han seguido liderando otras protestas y aunque no han tenido el mismo protagonismo, se han constituido como actores legitimados por las administraciones locales.

Durante los años ochenta, la elaboración de nuevos planes urbanísticos fue clave para la recuperación urbana de las ciudades heredadas de la Dictadura. La redacción de éstos fue planteada como una de las primeras acciones de los ayuntamientos democráticos a causa de la presión que ejercían los ciudadanos con sus reivindicaciones y las asociaciones de vecinos (AAVV) tuvieron un rol eminente en la fase de discusión pública, que no fue considerada por los ayuntamientos como una simple cuestión de trámite. En este sentido, se hicieron esfuerzos para difundir información y fomentar la participación de entidades y la ciudadania en general en conferencias, mesas redondas y sesiones informativas (Ferrer y Sabaté, 1999). Fue un urbanismo dialogado con la misma sociedad donde muchas veces las AAVV transmitían la voz de los ciudadanos. El movimiento vecinal, con su labor de oposición y sus reivindicaciones, ha configurado un legado que se estructura en tres aspectos: a) la revalorización del espacio público, de la calidad de vida y del barrio como espacio identitario; b) la exigencia de la democracia y de la participación en la elaboración de los planes, con un gobierno de proximidad; c) la toma de conciencia del derecho a la ciudadanía, el hecho de ser ciudadano y la necesidad de implicarse en el proceso de construcción de la ciudad (Alabart, 1998; Borja, 2003; Recio y Naya, 2004).

Con la proliferación de las plataformas de ciudadanos, a finales de los años noventa, la fragmentación de la representación de la ciudadanía es más signi- 
ficativa y a veces unos se presentan (o son reconocidos) como interlocutores válidos en tanto que asociación constituida frente a otros. La aparición de nuevas formas de organización al margen de las AAVV preexistentes se puede leer desde diferentes perspectivas: una falta de confianza de los ciudadanos movilizados respecto a las asociaciones de vecinos; una escasez de renovación interna de los activistas vecinales (con la consecuente falta de renovación de intereses, estructuras, perspectivas y temas); una moderación en las demandas por el proceso de institucionalización de las AAVV o simplemente debido a la mayor fragmentación y heterogeneidad en la actualidad de las organizaciones relacionadas con los movimientos urbanos, con objetivos y maneras de actuar diferentes.

En referencia a los conflictos, ambos grupos de actores, plataformas y asociaciones de vecinos, no siempre adoptan las mismas posiciones: a veces comparten ideas y se presentan como colaboradores y cómplices, otras discrepan y mantienen posiciones separadas, y en otras las AAVV se mantienen al margen del conflicto mientras la plataforma lidera la oposición. El distinto carácter temporal que asumen ambos grupos es otra de las principales diferencias: unos altamente efímeros, otros perviven desde hace treinta años. Las AAVV gozan, pues, de una posición de privilegio en la interlocución con la administración local ya que han logrado consolidarse en el escenario urbano y las relaciones que mantienen con los políticos son, en muchos casos, estrechas.

Un papel relevante en los conflictos lo tiene el de los "expertos críticos", a menudo académicos que, con el tiempo y gracias a su ayuda continua a los colectivos opositores, se convierten en un referente y se les otorga la etiqueta de expertos independientes. Así, las plataformas tienden a buscar a expertos críticos o contraexpertos, en tanto que se contraponen a los técnicos municipales (Lewanski, 1997), para contrastar con ellos de modo consultivo el proyecto elaborado por los técnicos municipales. Las plataformas se rodean de abogados, arquitectos, urbanistas, geógrafos, de los que recibir opiniones distintas, adquirir conocimientos técnicos nuevos y encontrar argumentos para rebatir el plan promovido por la administración ya que el carácter técnico-jurídico de las cuestiones urbanísticas da preferencia a aquellos actores que están capacitados para debatir. Muchas veces consiguen con argumentos bien fundados poner en duda las posiciones políticas o hasta incomodar a los mismos políticos (Bobbio, 1999).

Los medios de comunicación ejercen, por un lado, de canales a través de los cuales se dan a conocer los discursos de los actores que intervienen en el conflicto y, por otro, se establecen como actores propiamente dichos ya que pueden influenciar el desarrollo del conflicto y las estrategias de otros actores, tanto de las formaciones políticas como de los ciudadanos. En relación a los grupos opositores, los medios de comunicación ejercen una función de divulgación de sus reclamaciones y de las propuestas alternativas con un lenguaje menos técnico y más digerible hacia aquellos que desconocen la problemática. De esta manera ayudan a las movilizaciones a expandir su ámbito de influencia, y en ocasiones hasta a alargar el número de personas que les dan apoyo. De todas 
maneras, la misma actuación por parte de los medios puede tener consecuencias en el sentido opuesto. Tienden a simplificar el mensaje de los opositores (reduciéndolos al simple "no") y a concentrarse en aquellas acciones de protesta más espectaculares, prestando poca atención a los argumentos más de fondo y a los espacios de negociación. Medios de comunicación alternativos (periódicos, radios, portales web), así como la creación de blogs y la presencia en redes sociales de Internet se configuran como fuentes de información y comunicación más directas entre los opositores y los afectados, otros grupos activistas, los periodistas y la sociedad en general.

De todas formas, una vez estallado el conflicto los medios de comunicación ejercen un rol de trampolín. En el momento que sale a la luz pública que un determinado grupo de ciudadanos están descontentos con la actitud y decisiones de la administración, los políticos tienden a reaccionar rápidamente para evitar el boom que la continua presencia de las protestas en los medios de comunicación puede significar en términos de pérdida de confianza por parte de la ciudadanía, expansión del conflicto o pérdida de legitimidad política.

A menudo las administraciones públicas reconocen la falta de iniciativas para prevenir reacciones negativas por parte de la comunidad ante un proyecto urbanístico y reconocen que - por su parte - era difícil preverlas. Según Bobbio (1999) la infravaloración de la protesta es una de las características más persistentes de las administraciones ya que "tienden a preocuparse demasiado poco por los conflictos que pueden desencadenar sus iniciativas y se fían excesivamente de la bondad de las propias decisiones, de los estudios técnicos y de la fuerza de la ley" (Bobbio, 1999: 202-203). El alejamiento de los políticos respecto a los ciudadanos se traduce en los casos de conflicto en una actitud de infravalorar argumentos o propuestas que aportan los propios ciudadanos manifestando poca consistencia técnica para evitar que la alternativa tome fuerza (Bobbio, 1999) y en no considerar suficiente la representatividad de los ciudadanos organizados. De todos modos, estos comportamientos, en vez de desanimar a los ciudadanos, a menudo causan el efecto contrario: hacen radicalizar las posiciones y provocan que los grupos opositores tiendan a querer movilizar más población para mostrarse como un interlocutor más representativo. Así se inicia la protesta abierta, que es utilizada por parte de los ciudadanos como arma para sacudir y hacer reaccionar a los gobernantes locales y, en cierta manera, también a los técnicos municipales, los cuales suelen trabajar inmersos en la máquina de la administración. Otra estrategia habitual por parte de los oponentes es acudir a instancias de gobierno de nivel superior al local, ya sea regional, estatal o europeo.

En algunas ocasiones también se ha dado una utilización política de la protesta por parte de los partidos, a menudo aquellos que no gobiernan, o que están en coalición y quieren sacar un beneficio político de la protesta. Así, algunos de ellos optan por apoyar la protesta o fomentarla persiguiendo fines propios que no tienen porqué coincidir con los objetivos de la protesta. En estas ocasiones, los ciudadanos organizados también suelen utilizar a aquellos partidos políticos que les ofrecen apoyo como puerta de acceso al escenario político 
aprovechándose de las divisiones entre las elites. Como resultado, se produce una utilización recíproca donde ambos actores reciben compensaciones.

\section{EL CONFLICTO DE CAN RICART}

En tiempos recientes se han sucedido importantes conflictos urbanísticos en Barcelona. Entre ellos cabe destacar el conflicto del "Forat de la Vergonya" (Verger y Mas, 2004; Xalabarder 2007, 2006, 2005a) en pleno corazón de la ciudad, la controvertida remodelación de la Plaza Lesseps, (Herrero, 2004, 2005; González, 2006; Sau, 2009) la recalificación del Mini-estadio del Futbol Club Barcelona (Carreras, 2006a; Carbó, 2008, 2007), la transformación de los antiguos cuarteles en Sant Andreu (Xalabarder, 2005b; Carreras, 2006b), la discusión entorno a la conservación de Can Ricart en el Poblenou, el derribo de las casas baratas en Bon Pastor (Xalabarder, 2005c, 2008), la construcción del Hotel Vela en la Barceloneta, etc. En todos estos conflictos, que surgen de la aprobación de proyectos puntuales - ya sean de renovación del espacio público o de regeneración de un barrio - que han conllevado alguna modificación en el Plan General Metropolitano de Barcelona (aprobado en 1976), se reflejan las características anteriormente mencionadas, si bien cada uno tiene obviamente sus propias particularidades. A continuación, para ejemplificar con más detalle los aspectos abordados en los anteriores apartados, nos adentraremos en el conflicto entorno a la preservación y los usos del recinto industrial de Can Ricart en el barrio del Poblenou. Aunque son muchos los conflictos urbanísticos en los últimos años, abordaremos este en profundidad por estar enmarcado en uno de los principales proyectos estratégicos de la ciudad (el plan22@bcn), por su complejidad de estrategias, discursos y de actores, así como por los resultados conseguidos.

El recinto industrial, proyectado entre 1852 y 1853 , fue una de las primeras industrias de estampación mecánica de Cataluña así como una de las manufacturas más importantes del Barcelona. En sus inicios albergó una fábrica de estampaciones de algodón y a partir de los años 20 se convirtió en un parque industrial acognó multiplicidad de industrias y talleres. A partir de los años 90, se alojaron además talleres de artistas, como por ejemplo Hangar, equipamiento de producción audiovisual gestionado inicialmente por la asociación de artistas audiovisuales y cofinanciado por el municipio. A pesar de las reparcelaciones, era de los pocos recintos industriales de su época que con el tiempo se había mantenido íntegro.

Este recinto fue incluido en el plan de renovación de las áreas industriales del Poblenou, el Plan 22@bcn, que afectaba a 198,26 hectáreas. Aprobado en el año 2000, este plan tiene como objetivo desarrollar y atraer nuevas actividades y empresas ligadas a la "economía del conocimiento" (formación, investigación, producción audiovisual, servicios a empresas, actividades relacionadas con las TIC y la cultura, etc.). Con ese objetivo se prevé aumentar la densidad y la 
diversidad de usos de las antiguas zonas industriales. Un año después se aprobó el tercer de los planes especiales de iniciativa pública incluidos en el plan22@ bcn, el del Parc Central, que abarca principalmente al recinto de Can Ricart. Este plan, que posteriormente fue modificado, mantenía solamente algunos elementos aislados del recinto: la chimenea, la torre del reloj (torre principal de la fábrica) y dos naves. Esto suponía el derribo de buena parte del recinto y su partición con la apertura de una nueva calle para seguir con la trama característica del Ensanche de Cerdà. La mayor parte del recinto se calificó como suelo 22@1o que posibilitaba un amplio abanico de usos (residencial, equipamientos, actividades económicas, etc), aunque mayormente significaba la construcción de oficinas.

En el momento de su aprobación, en Can Ricart había en funcionamiento un bar, 34 empresas que ocupaban a unos 250 trabajadores y dos espacios dedicados a la creación artística (Hangar y Nau-21), todos en régimen de alquiler. Cuando las empresas allí ubicadas vieron finalizar sus contratos, crearon en 2005 la "Asociación de trabajadores y empresarios de Can Ricart" para reclamar indemnizaciones justas para todos los negocios, independientemente del tipo de contrato de alquiler, que les permitieran afrontar los costes de relocalización. La implicación de más de una cuarentena de entidades y colectivos culturales, educativos, vecinales y juveniles de la ciudad provocó un cambio de escala, de un conflicto entre particulares pasó a ser un problema de la ciudad. Así se creó la plataforma "Salvem Can Ricart" que unió las reivindicaciones de conservación del recinto por razones históricas, identitarias y arquitectónicas con las reivindicaciones de les empresarios y trabajadores para mantener sus actividades.

Entre los colectivos que formaron la plataforma, podemos destacar la asociación de vecinos del Poblenou, el Fórum Ribera Besos y especialmente el grupo de patrimonio industrial del Fórum, formado por líderes vecinales y académicos. Este grupo tuvo un papel fundamental a lo largo del proceso, tanto por el activismo de sus miembros como por la capacidad de investigación-acción que posibilitó la elaboración de informes y proyectos alternativos dando solidez a las reivindicaciones de la Plataforma (Grup de Patrimoni Industrial del Fòrum Ribera Besòs, 2005, 2006a, 2006b, 2006c; Clarós et al., 2006). Otro colectivo muy activo e innovador fue el de los artistas, con Hangar y el grupo alrededor del taller de Can Font - Nau21. Este último elaboró sus propias propuestas para convertir Can Ricart en un espacio de innovación urbana de dominio público que combinara espacios creativos, industriales y de promoción del patrimonio (Nau 21, 2006). La asociación de empresarios y trabajadores de can Ricart trabajó activamente dentro de la plataforma así como diferentes entidades culturales y juveniles del Poblenou.

Las primeras acciones de la Plataforma fueron dar apoyo a las empresas amenazadas e intentar evitar su desalojo. Paralelamente se realizaron visitas guiadas, asambleas de vecinos, festivales culturales, manifestaciones, etc., para dar a conocer Can Ricart y su problemática al barrio y a la ciudad. En ese 
momento Can Ricart se convirtió en un símbolo de resistencia vecinal creativa frente a las presiones especulativas y a la planificación urbana sin participación. La dimensión que tomó el conflicto propició que el Ayuntamiento mediara entre el propietario del recinto y los negocios afectados y finalmente, con un aumento de las indemnizaciones, las empresas abandonaron progresivamente el recinto. Así, a finales de junio del 2006, cerró la última empresa que quedaba en el recinto. Entre las que no cerraron y se pudieron relocalizar, solamente una continuó en el mismo Poblenou (Grup d'Etnologia dels Espais Públics de l'Institut Català d'Antropologia, 2006). A partir de ese momento únicamente mantuvo su actividad Hangar, debido a que los espacios donde se ubicaba pertenecen al Ayuntamiento.

Como resultado de las posturas inmovilistas del Departamento de Urbanismo del Ayuntamiento, la Plataforma abrió nuevos frentes de incidencia hacia niveles superiores de gobierno que finalmente tuvieron importantes impactos en el planeamiento urbano. El primer frente fue la interlocución con los partidos políticos con representación en el Parlamento catalán para presentar una iniciativa legislativa para preservar el patrimonio y la memoria histórica en todo Poblenou (Clarós et al., 2005). La segunda fue el inicio de los trámites en el Departamento de Cultura de la Generalitat de Cataluña para declarar el recinto de Can Ricart Bien Cultural de Interés Nacional (BCIN) y asegurar su protección completa. Además, en este periodo se realizaron estudios en profundidad y se elaboró un planeamiento alternativo al municipal que, manteniendo las plusvalías y los derechos adquiridos por el propietario, permitía conservar la fábrica íntegra (Grup de Patrimoni Industrial del Fòrum Ribera Besòs, 2005). Una vez asumida la pérdida de las antiguas actividades empresariales, se propusieron también nuevos usos para el recinto, como la ubicación de equipamientos del barrio (guarderías, centros de barrio), equipamientos de ciudad (museo del trabajo), viviendas de protección social y espacios dedicados a talleres de artistas y centros de investigación. Se proponía así una combinación de usos productivos, culturales y ciudadanos de relevancia tanto local como global, con la idea de convertir el espacio en un nuevo polo de centralidad urbana ciudadana (Grup de Patrimoni Industrial del Fòrum Ribera Besòs, 2006c).

Como reacción a las presiones de la plataforma, el Ayuntamiento anunció a principios de abril del 2007 un nuevo plan urbanístico para Can Ricart más respetuoso con el patrimonio industrial. Pocas horas más tarde, un incendio destruyó una nave y afectó la parte más significativa de la fábrica: la torre del reloj, incendiando dos piezas de reciente adquisición municipal. Este episodio contribuyó a la aceleración del deterioro del recinto, un proceso que se precipitó después de la expulsión de las empresas. La Plataforma "Salvem Can Ricart", conjuntamente con otros colectivos del barrio, además de pedir responsabilidades en relación al incendio, rechazaron el nuevo plan y presentaron alegaciones. En ese momento, también entró en escena un nuevo actor en el conflicto: la Makabra. Este colectivo alternativo, especializado en las artes circenses y desalojado pocos días antes de una fabrica cercana, okupó Can Ricart a finales del 
2006. Después de resistir un cerco policial de 34 horas, consiguieron evitar el desalojo urgente y la plataforma reaccionó dando apoyo a la okupación. Los talleres de artistas que progresivamente se habían instalado en el Poblenou durante los años 90 estaban siendo expulsados con el desarrollo del plan 22@bcn (MartíCosta y Pradel, en prensa). Finalmente, las presiones políticas y del propietario forzaron su desalojo 11 días más tarde, antes de que se realizaran las primeras actividades abiertas al público. Tanto el incendio como la ocupación recibieron una gran atención por parte de los medios de comunicación, lo que aumentó la sensación de conflicto entorno al recinto, si bien también difuminó las propuestas y acciones realizadas por la plataforma.

Poco a poco el perseverante trabajo del movimiento urbano empezó a dar sus frutos. Como resultado de la iniciativa parlamentaria, en diciembre del 2006 se aprobó un plan de patrimonio para el Poblenou donde se incluyeron 68 nuevos elementos y se amplió la protección de diferentes conjuntos partimoniales (Ajuntament de Barcelona, 2006). A pesar del avance, en ese momento ya habían sido destruidas por las excavadoras fábricas importantes (como por ejemplo Extractos Tánicos), el grado de protección de muchos elementos continuaba siendo bajo (solamente protección documental), tenía poco en cuenta el paisaje en su conjunto y no significaba modificaciones importantes en relación a Can Ricart, a pesar de que pasaba a ser declarado Bien Cultural de Interés Local (Tatjer, 2008). Pero el éxito más significativo del movimiento vendrá de la declaración de Bien Cultural de Interés Nacional en categoría de conjunto histórico por parte del Departamento de Cultura de la Generalitat de Cataluña. Eso implicó la suspensión de las licencias de obras vigentes y que las nuevas tuvieran que ser aprobadas por la comisión de patrimonio cultural de la Generalitat.

Como resultado, mientras que en el terreno de la protección del patrimonio se obtuvieron avances significativos, en relación a los usos del espacio el resultado fue menos fructífero. A excepción de Hangar, todas las actividades precedentes fueron expulsadas y el recinto entró en proceso de degradación: "Con el tiempo se ha producido una terrible paradoja: cuando el 2005 se iba a derribar según el plan especial, la fábrica estaba de pie y se trabajaba en ella, ahora, que parece que se salva la mayor parte, está en ruina, destrozada por dos incendios, y todo es una trinchera llena de muros, zanjas y garitas" (Montaner, 2007). Tampoco los usos aprobados en el nuevo plan municipal (lofts, museo de los idiomas, oficinas, etc.) coinciden con los propuestos por el movimiento.

\section{REFLEXIONES FINALES}

La transformación urbana de la ciudad de Barcelona durante las últimas dos décadas se ha llevado a cabo a través de proyectos urbanos que han implicado la introducción de modificaciones puntuales en el Plan General Metropolitano, vigente desde 1976. Así, se ha evitado iniciar un proceso de revisión del planeamiento urbanístico de la ciudad (y del área metropolitana) y se ha optado 
por mecanismos administrativos e instrumentos de bon planificación más ágiles y flexibles. Como el caso de Can Ricart i el Plan 22@, ejemplifican (pero al igual que muchos otros proyectos), el futuro de la ciudad no ha sido reflexionado y debatido de forma global sino que se ha actuado de forma fragmentaria, repensando distintas áreas de la ciudad de forma individual.

En este sentido, el plan especial ha resultado un instrumento útil para actuar de forma concreta y aislada en la trama urbana. Además, a diferencia del proceso de tramitación de los planes generales, el de los planes especiales cuenta con un sólo período obligatorio de información pública, de manera que la tramitación de los proyectos urbanos no ha facilitado el debate y la participación de la ciudadanía en su elaboración sino más bien ha resultado apetecible por agentes privados y el Ayuntamiento, interesados en una actuación urbanística rápida. Por lo tanto, la ausencia de procesos de planificación que se interesaran para debatir con todos los agentes urbanos (responsables políticos, propietarios del suelo, promotores inmobiliarios, constructores, técnicos, entidades cívicas, asociaciones de vecinos, etc.) sus prioridades e intereses ha tendido a desencadenar el estallido de conflictos urbanísticos ya que la ciudadanía ha percibido que estos proyectos urbanos eran diseñados y aprobados sin que ellos (los usuarios finales de la ciudad) hubieran tenido la oportunidad de expresar su opinión.

Así pues, la espiral competitiva en la cual está inmersa la ciudad ha conllevado una actuación urbanística flexible y rápida que bien poco se ha preocupado para reflexionar sobre la conveniencia de los proyectos propuestos sino más bien para ejecutarlos cuanto antes. En consecuencia, la poca atención prestada para conocer (a través de amplios procesos de participación) los deseos y necesidades de aquellos que debían acoger los proyectos ha representado la semilla para que en el proceso de aprobación o ejecución de los planes la ciudadanía se rebelara ante una actuación urbanística que consideraba foránea e impuesta.

En relación a la organización del movimiento urbano, el caso expuesto muestra la heterogeneidad de colectivos incluidos en el conflicto, a veces actuando de forma conjunta bajo el paraguas de una plataforma, a veces actuando de forma autónoma. La creación de la plataforma permitió sumar esfuerzos agrupando un amplio espectro de organizaciones en su seno con un grado de implicación diversa, que de otra forma sería difícil aglutinar. Aunque la asociación de vecinos del Poblenou fue uno de los actores centrales en la constitución y dinamización de la plataforma, su trabajo se realizó en continua interlocución y cooperación con otros grupos. Entre estos otros, en el caso expuesto toman nuevos protagonismos los squatters, grupos de artistas o activistas relacionados con el ciberactivismo. Si bien el trabajo en red permitió la generación de alianzas variables que fortalecieron y legitimaron el movimiento, a veces también fue fruto de tensiones y separaciones por la confluencia de intereses y de backgrounds diferentes, tanto dentro como fuera de la plataforma.

En el caso de Can Ricart, numerosos profesionales de diferentes ámbitos han dedicado tiempo e ideas, conjuntamente con miembros y simpatizantes de 
la plataforma, a fundamentar las críticas al plan. Se han llegado a realizar hasta nueve estudios diferentes sobre la fábrica y sus alternativas que incluyen una amplia documentación de planos, mapas, dibujos y maquetas; generándose una amplia documentación (Grup de Patrimoni Industrial del Fòrum Ribera Besòs, 2006c), sin tener en cuenta las decenas de artículos de opinión favorables al movimiento que han aparecido en los medios de comunicación. La construcción colectiva de conocimiento ha sido sin duda uno de los puntos fuertes de la movilización. A partir de un enfoque interdisciplinario se ha combinado la incorporación de saberes provenientes del urbanismo, la historia, la arquitectura, la ingeniería y la sociología entre otros para dar forma a las ideas de la plataforma y orientar las nuevas acciones. Especialmente relevante ha sido el proceso de socialización y difusión de conocimiento que se ha producido entorno a aspectos históricos y arquitectónicos entre los vecinos del Poblenou, lo que ha permitido la autoformación de activistas y simpatizantes, estimulando el debate de ideas, habilidades y recursos tanto dentro de como fuera del movimiento. Al mismo tiempo, la combinación de la reflexión con la acción ha permitido ir más allá de la defensa conservadora de un espacio concreto. Se han aportado nuevas ideas sobre la construcción de la ciudad, tanto en los aspectos procedimentales (una profundización democrática de los procesos de planificación), como en aquellos más substantivos: nuevas formas de centralidad urbana que no sean sólo a partir de centros comerciales o de oficinas, una vinculación más estrecha entre el habitar y la planificación o el reto de gobernar incorporando ideas innovadoras sin banalizarlas.

Por lo que se refiere a la relación con la administración, se ha ilustrado que el actual contexto institucional ofrece grietas y posibilidades si se saben utilizar las discrepancias entre elites y los diferentes organismos públicos, tanto a nivel horizontal (entre coaliciones de actores, como por ejemplo partidos que forman un mismo gobierno) como a nivel vertical, entre diferentes niveles de gobierno (local, autonómico, estatal supraestatal). En el caso de Can Ricart para tratar de modificar una decisión urbanística local, a parte de la presión e interlocución directa con el Ayuntamiento, se utilizó el bypass del Parlamento catalán por un lado y el de la política cultural de la Generalitat por el otro. Esto evidentemente se combinó con un repertorio de acciones colectivas variadas encaminadas a la protesta (manifestaciones) y a la sensibilización y difusión (charlas, web, publicaciones, performance, actuaciones, etc.). Cabe destacar la innovación en nuevas formas de involucrar a los ciudadanos y la autofinanciación del movimiento, como por ejemplo en la campaña de adquisición de acciones de la ficticia "Compañía Ciudadana de Can Ricart”.

También hemos mostrado la diversidad de viejas y nuevas temáticas puestas sobre la mesa a partir del conflicto urbanístico. Las movilizaciones entorno a Can Ricart no sólo han luchado para modificar el proyecto urbano ideado para su barrio desde el Ayuntamiento, sino que han elaborado un amplio repertorio argumental que reivindicaba tanto la necesidad de nuevos equipamientos colectivos y de creación como la urgencia para establecer políticas de preservación 
del patrimonio, pero también advertía de los impactos en términos de gentrificación que se podían derivar del proyecto y denunciaba los intereses de especulación de determinados agentes urbanos; en definitiva reclamaba mejoras en la calidad democrática de la toma de decisiones en las cuestiones urbanísticas.

\section{BIBLIOGRAFÍA}

Ajuntament de Barcelona (2009) Síntesi de la diagnosi i línies de treball per a l'elaboració del Pla Director Municipal de Participació Ciudadana 2010-2015. Ajuntament de Barcelona, Barcelona.

Ajuntament de Barcelona (2006) Modificació del pla especial de protecció del patrimoni arquitectònic historicoaristic de la ciutat de Barcelona. Districte de Sant Martí. Patrimoni industrial del Poblenou.22@bcn. Ajuntament de Barcelona, Barcelona.

Ajuntament de Barcelona (2001) Pla especial del pla de reforma interior del sector Parc Central de la MPGM per a la renovació de les àrees industrials del Poblenou-Districte d'activitats 22@, bcn -. Sector d'Urbanisme. Ajuntament de Barcelona, Barcelona.

Ajuntament de Barcelona (2000) Modificació del PGM per les àrees industrials del Poblenou-Districte d'activitats 22@-. Text Refós. Sector d'Urbanisme. Ajuntament de Barcelona, Barcelona.

Alabart A (1998) Els moviments socials urbans a Catalunya. Revista Catalana de Sociologia, 7: 928.

Alexander C (1976) Urbanismo y participación. Gustavo Gili, Barcelona.

Alfama E, Casademunt À, Coll G, Cruz H, Martí M (2007) Per una nova cultura del territori. Icaria, Barcelona.

Blanco I, y Gomà R (2002) Proximidad y participación: marco conceptual y presentación de experiencias. In Blanco I, Gomà R (coords.) Gobiernos locales y redes participativas. Ariel, Barcelona: 21-41.

Bobbio L (1999) Un processo equo per una localizzazione equa. In Bobbio L, Zeppetella A (a cura de) Perché proprio qui? Grandi opere e opposizioni locali. Franco Angeli, Milano: 185-223.

Borja J (2003) La ciudad conquistada. Alianza Editorial, Madrid.

Brenner N (1999) Globalisation as reterritorialisation: the re-escaling of urban governance in the European Union. Urban Studies, 36(3): 431-451.

Camagni R (2003) Piano strategico, capitale relazionale e community governance. In Pugliese T, y Spaziante A (a cura de) Pianificazione strategica per le città: riflessioni dalle pratiche. Franco Angeli, Milano: 79-100.

Carbó R (2008) Transformació urbana dels entorns del Camp Nou (Barcelona). In Tarroja À, Castañer M, y Mercadé M (dirs.) Anuari Territorial de Catalunya 2007. Societat Catalana d'Ordenació del Territori, Barcelona: 474-475.

Carbó R (2007) Transformació urbana de l'entorn del Camp Nou (Barcelona). In Tarroja À, Castañer M, y Mercadé M (dirs.) Anuari Territorial de Catalunya 2006. Societat Catalana d'Ordenació del Territori, Barcelona: 438-439.

Carreras J (2006a) Transformació urbana de l'entorn del Camp Nou (Barcelona). In Tarroja À, Castañer M, y Mercadé M (dirs.) Anuari Territorial de Catalunya 2005. Societat Catalana d'Ordenació del Territori, Barcelona: 412-413.

Carreras J (2006b) Transformació urbana de les Casernes de Sant Andreu (Barcelona). In Tarroja À, Castañer M, y Mercadé M (dirs.) Anuari Territorial de Catalunya 2005. Societat Catalana d'Ordenació del Territori, Barcelona: 414.

Castells M (1997) La era de la información. Economía, sociedad y cultura. La sociedad red. Alianza editorial, Madrid.

Castells M (1977) Movimientos sociales urbanos. Siglo XXI, Madrid.

Castells M (1976) La Cuestión Urbana. Siglo XXI, Madrid.

Clarós S, et al. (2006) Can Ricart, proposta d'intervenció. Revista Bibliográfica de Geografía y Ciencias Sociales, XI(630). 
Clarós S, et al. (2005) Proposta de pla de patrimoni industrial de Barcelona. Revista Bibliográfica de Geografía y Ciencias Sociales, X(581).

Cruz H (2006) Strategic urban planning in Barcelona and Venice, an analysis of the methodological process. In Carlow V M, y Speller C J M (eds.) Urban management in Europe. Towards a sustainable development. Erasmus University Rotterdam, Rotterdam: 159-186.

Curti F, y Gibelli M C (1996) (a cura de) Pianificazione strategica e gestione dello sviluppo urbano. Alinea, Firenze.

Davidoff P (1965) Advocacy and pluralism in planning. In LeGates R T, Stout F (eds.) (1998) The city reader. Routledge, London: 422-432.

Dear M (1992) Understanding and overcoming the NIMBY syndrome. Journal of the American Planning Association, 58(3): 288-300.

della Porta D (a cura de) (2004) Comitati di cittadini e democrazia urbana. Rubbettino, Catanzaro.

della Porta D, Diani M (2004) Movimenti senza protesta?L'ambientalismo in Italia. Il Mulino, Boloña.

Esteban J (1999) Pla urbanístic versus pla estratègic. Quaderns de la Societat Catalana d'Ordenació del Territori, 9: 87-101.

Faggi P, y Turco A (a cura de) (2001) Conflitti ambientali. Genesi, sviluppo, gestione. Unicolpi, Milán.

Ferrer A, y Sabater J (1999) L'urbanisme municipal. In Nel-lo O (coord.) 20 anys d'ajuntaments democràtics. Federació de Municipis de Catalunya, Barcelona: 117-160.

Font A (2003) Planeamiento urbanístico: de la controversia a la renovación. Diputació de Barcelona, Barcelona.

Font J (2009) Experiències de participació en els municipis catalans. Un mapa analític. Generalitat de Catalunya, Barcelona.

Forn M de, y Pascual J M (1995) La planificació estratègica territorial. Aplicació als municipis. Diputació de Barcelona, Barcelona.

Friedman J (1992) Empowerment the politics of alternative development. Blackwell, Oxford.

González I (2006) Hacia una nueva sonoridad democrática. Los procesos no estructurados de participación ciudadana. In Tarroja À y Camagni R (coords.) Una nueva cultura del territorio. Diputació de Barcelona, Barcelona: 469-476.

Grup de Patrimoni Industrial del Fòrum Ribera Besòs (2005) Can Ricart - Parc Central. Nou Projecte. Fòrum Ribera Besòs, Barcelona.

Grup de Patrimoni Industrial del Fòrum Ribera Besòs (2006a) Proposta de criteris d'intervenció, Fòrum Ribera Besòs, Barcelona. [Accedido el 13 de julio de 2007]. http://www.salvemcanricart.org

Grup de Patrimoni Industrial del Fòrum Ribera Besòs (2006b) Can Ricart, recinte o fragments? Tractament del patrimoni, inserció urbana i reutilització del complex fabril. Fòrum Ribera Besòs, Barcelona. [Accedido el 13 de julio de 2007]. http://www.salvemcanricart.org

Grup De Patrimoni Industrial del Fòrum Ribera Besòs (ed) (2006c) Can Ricart. Patrimoni, innovació $i$ ciutadania. Fòrum Ribera Besòs, Barcelona.

Grup d'Etnologia dels Espais Públics de l'Institut Català d'Antropologia (2006) Víctimes del 22@... les empreses de Can Ricart. [Accedido el 13 de julio de 2007]. http://barcelona.indymedia. org/usermedia/image/12/large/victimes22@_can_ricart_s.jpg

Healey P (1997) Collaborative planning. Shaping places in fragmented societies. Macmillan, Hampshire.

Herrero M (2005) Millora urbana de la Plaça Lesseps (Barcelona). In Tarroja À (dir.) Anuari Territorial de Catalunya 2004. Societat Catalana d'Ordenació del Territori, Barcelona: 247-248.

Herrero M (2004) La Plaça Lesseps. In Esteban J, y Tarroja À (dirs.) Anuari Territorial de Catalunya 2003. Diputació de Barcelona y Societat Catalana d'Ordenació del Territori, Barcelona: 260-261.

Harvey D (1990) The condition of postmodernity. Basil Blackwell, Oxford.

Indovina F (2005) Governare la città con l'urbanistica. Maggioli, Santarcangelo di Romagna.

Köhler B, y Wissen M (2003) Glocalizing protest: urban conflicts and global social movements. International Journal of Urban and Regional Research, 27(4): 942-51.

Lagomarsino L (2002) Progetto e partecipazione. In Cristoforetti G, Ghiara H (a cura de) Progetto, conflitti e territorio. Alinea Ed, Florencia: 17-24. 
Lewanski R (1997) Governare l'ambiente. Attori e processi della politica ambientale: interessi in gioco, slide, nuove strategie. Il Mulino, Boloña.

Logan J, y Molotoch H (1987) Urban fortunes: the political economy of place. University of California Press, Berkeley y Los Angeles.

Martí-Costa M, y Bonet J (2008a) Los movimientos urbanos hoy: de la identidad a la glocalidad. Scripta Nova. XII, 270 (121).

Martí-Costa M, y Bonet J (2008b) Planning from below in Barcelona. In Shaw K, y Portter L (ed.) Whose urban regeneration? An international comparaison. Routledge, Oxford: 118-128

Martí-Costa M, y Pradel M (en prensa) City of knowledge agains urban creativity? Creative workshops and urban regeneration in Barcelona. European Urban and Regional Studies.

Montaner J M (2007) Can Ricart, estat de la qüestió. El País (28 diciembre del 2007).

Nau 21 (2006) Can Ricart. Un espai urbà pel s.XXI. Un patrimoni viu: obert, productiu, creatiu. [Accedido el 15 de enero de 2010] http://nau21.net

Navarro F (1999) La gestió els plans. Quaderns de la Societat Catalana d'Ordenació del Territori, 9: $77-82$

$\mathrm{Nel} \cdot 10 \mathrm{O}(2003)$ Els conflictes territorials a Catalunya. Orígens, dinàmica i alternatives. In Nel·lo O (ed.) Aquí, no! Els conflictes territorials a Catalunya. Empúries, Barcelona: 11-67.

Recio A, y Naya A (2004) Movimiento vecinal: claroscuros de una lucha necesaria. Mientras tanto, 91-92: 63-82.

Sancassiani W (2005) Gestire i processi deliberativi: problemi e soluzioni. In Pellizzoni L (a cura de) La deliberazione pubblica. Meltemi, Roma: 205-227.

Sau, E (2009) Millora urbana de la Plaça Lesseps (Barcelona). Anuari Territorial de Catalunya 2009. [Accedido el 30 de junio de 2010] http://territori.scot.cat/cat/notices/2009/11/millora_urbana_ de_la_plaCa_lesseps_barcelona_1525.php

Subirats J (2006) La gobernabilidad de las políticas territoriales. Formulación participativa y gestión concertada. In Tarroja À, Camagni R (coords.) Una nueva cultura del territorio. Criterios sociales y ambientales en las políticas y el gobierno del territorio. Diputació de Barcelona, Barcelona: 398-408.

Swyngedouw E (1997) Neither global nor local. "Glocalization" and the politics of the scale. In Cox K R (ed.) Spaces of globalization. Reasserting the power of the local. The Guilford Press, New York \& London.

Tatjer M (2008) El patrimonio industrial de Barcelona entre la destrucción y la conservación, 19992008. Scripta Nova, XII, 270 (140).

Verger T y Mas M (2004) Un forat de la vergonya al Casc Antic de Barcelona. In Unió Temporal d'Escribes (ed.) Barcelona marca registrada. Virus, Barcelona: 308-317

Xalabarder M (2008) Transformació urbana del Bon Pastor (Barcelona). In Tarroja À, Castañer M, y Mercadé M (dirs.) Anuari Territorial de Catalunya 2007. Societat Catalana d'Ordenació dey Territori, Barcelona: 468-469.

Xalabarder M (2007) Transformació urbana del Pou de la Figuera (Barcelona). In Tarroja À, Castañer M, y Mercadé M (dirs.) Anuari Territorial de Catalunya 2006. Societat Catalana d'Ordenació del Territori, Barcelona: 448-449.

Xalabarder M (2006) Transformació urbana de Santa Caterina (Barcelona). In Tarroja À, Castañer M, y Mercadé M (dirs.) Anuari Territorial de Catalunya 2005. Societat Catalana d'Ordenació del Territori, Barcelona: 419-420.

Xalabarder M (2005a) Transformació urbana de Santa Caterina (Barcelona). In Tarroja À (dir.) Anuari Territorial de Catalunya 2004. Societat Catalana d'Ordenació del Territori, Barcelona: 392-393.

Xalabarder M (2005b) Transformació urbana de les Casernes de Sant Andreu (Barcelona). In Tarroja À (dir.) Anuari Territorial de Catalunya 2004. Societat Catalana d'Ordenació del Territori, Barcelona: 386.

Xalabarder M (2005c) Transformació urbana del Bon Pastor (Barcelona). In Tarroja À (dir.) Anuari Territorial de Catalunya 2004. Societat Catalana d'Ordenació del Territori, Barcelona: 394395. 